\title{
Antiproton Confinement in a Penning-Ioffe Trap for Antihydrogen
}

\author{
G. Gabrielse, ${ }^{1, *}$ P. Larochelle, ${ }^{1}$ D. Le Sage, ${ }^{1}$ B. Levitt, ${ }^{1}$ W. S. Kolthammer, ${ }^{1}$ I. Kuljanishvili, ${ }^{1}$ R. McConnell, ${ }^{1}$ J. Wrubel, ${ }^{1}$ \\ F. M. Esser, ${ }^{2}$ H. Glückler, ${ }^{2}$ D. Grzonka, ${ }^{3}$ G. Hansen, ${ }^{2}$ S. Martin, ${ }^{3}$ W. Oelert, ${ }^{3}$ J. Schillings, ${ }^{2}$ M. Schmitt, ${ }^{2}$ T. Sefzick ${ }^{3}$ \\ H. Soltner, ${ }^{2}$ Z. Zhang, ${ }^{3}$ D. Comeau, ${ }^{4}$ M. C. George, ${ }^{4}$ E. A. Hessels, ${ }^{4}$ C. H. Storry,${ }^{4}$ M. Weel,,${ }^{4}$ A. Speck, ${ }^{5}$ F. Nillius, ${ }^{6}$ \\ J. Walz, ${ }^{6}$ and T. W. Hänsch ${ }^{7,8}$
}

(ATRAP Collaboration)

${ }^{1}$ Department of Physics, Harvard University, Cambridge, Massachusetts 02138, USA

${ }^{2} Z A T$, Forschungszentrum Jülich GmbH, 52425 Jülich, Germany

${ }^{3} I K P$, Forschungszentrum Jülich GmbH, 52425 Jülich, Germany

${ }^{4}$ Department of Physics and Astronomy, York University, Toronto, Ontario M3J 1P3, Canada

${ }^{5}$ Rowland Institute at Harvard, Harvard University, Cambridge, Massachusetts 02142, USA

${ }^{6}$ Institut fur Physik, Johannes Gutenberg-Universität, D-55099 Mainz, Germany

${ }^{7}$ Max-Planck-Institut für Quantenoptik, Hans-Kopfermann-Strasse 1, 85748 Garching, Germany

${ }^{8}$ Ludwig-Maximilians-Universität München, Schellingstrasse 4/III, 80799 München, Germany

(Received 19 December 2006; published 14 March 2007)

\begin{abstract}
Antiprotons $(\bar{p})$ remain confined in a Penning trap, in sufficient numbers to form antihydrogen $(\overline{\mathrm{H}})$ atoms via charge exchange, when the radial field of a quadrupole Ioffe trap is added. This first demonstration with $\bar{p}$ suggests that quadrupole Ioffe traps can be superimposed upon $\bar{p}$ and $e^{+}$traps to attempt the capture of $\overline{\mathrm{H}}$ atoms as they form, contrary to conclusions of previous analyses.
\end{abstract}

DOI: 10.1103/PhysRevLett.98.113002

A long-term goal for $\overline{\mathrm{H}}$ experiments is confining $\overline{\mathrm{H}}$ in a magnetic trap for precise laser spectroscopy [1] - to compare $\overline{\mathrm{H}}$ and $\mathrm{H}$ as a test for violations of $C P T$ and Lorentz invariance [2] and for possible differences in the gravitational force on antimatter and matter $[3,4]$. These objectives were recently reviewed [5], along with the two methods that produce $\overline{\mathrm{H}}$ atoms - using a nested Penning trap [6-10] and using laser-controlled charge exchange $[11,12]$. The simplest approach is to superimpose the magnetic gradient needed to trap $\overline{\mathrm{H}}$ atoms upon the uniform magnetic field used to store the $\bar{p}$ and $e^{+}$from which $\overline{\mathrm{H}}$ will form. The quadrupole Ioffe traps that confined $\mathrm{H}$ atoms [13] for extremely precise laser spectroscopy [14] should confine similarly cold $\overline{\mathrm{H}}$ atoms. However, three Letters in this journal expressed concern as to whether the radial field of such magnetic traps would prevent $\bar{p}$ and $e^{+}$from being trapped long enough to produce $\overline{\mathrm{H}}$ atoms [15-17]. The last of these claimed that the radial field of such magnetic traps would keep $\overline{\mathrm{H}}$ from being produced by any known $\overline{\mathrm{H}}$ formation mechanism [17]. These studies focused upon radial Ioffe fields, perpendicular to the axial magnetic field of the Penning trap, assuming that axial Ioffe fields added to trap $\overline{\mathrm{H}}$ could always be made small at the location of the trapped charges.

We demonstrate here the stable confinement of $\bar{p}$ in a Penning trap, when the radial magnetic field of a quadrupole Ioffe trap destroys the axial symmetry. This first experimental study of such $\bar{p}$ stability is facilitated by the near-unit efficiency with which annihilation pions reveal $\bar{p}$ losses. More $\bar{p}$ remain confined in our Penning trap apparatus (Fig. 1) than are needed to use ATRAP's laser-
PACS numbers: 32.80.Pj, 36.10.-k, 41.20.-q, 41.90.+e

controlled charge-exchange method to produce $\overline{\mathrm{H}}[12]$. The feasibility of also keeping the needed $e^{+}$confined in this environment is demonstrated with electrons. Ioffe quadrupole traps thus seem to have a role in $\overline{\mathrm{H}}$ experiments, despite contrary claims, though much remains to be opti-

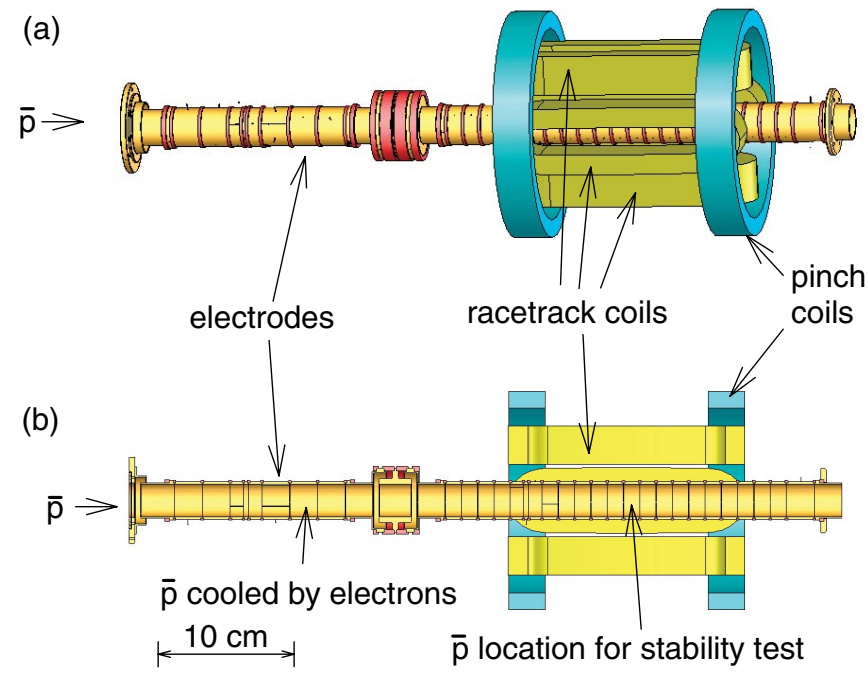

FIG. 1 (color). (a) Outside and (b) cutaway views of a Penning-Ioffe apparatus. Many cylindrical ring electrodes can be biased to form Penning traps for antiprotons, positrons, and electrons. An external 1 Tesla bias field, directed along the central symmetry axis of these electrodes, is produced by a large external solenoid (not shown). Two pinch coils add a gradient to the axial field. The radial quadrupole Ioffe field is produced by four racetrack coils. 
mized and studied when $\bar{p}$ eventually become available again.

There is a basic reason to fear charged particle loss from a Penning or Penning-Malmberg trap when the radial magnetic field of a Ioffe trap is added [15]. A confinement theorem [18] prevents radial particle loss from a cylindrically symmetric Penning trap-a spatially uniform magnetic field $B_{0} \hat{\mathbf{z}}$ and an electrostatic quadrupole potential $V \propto\left[z^{2}-\left(x^{2}+y^{2}\right) / 2\right]$. However, the axial symmetry and the guaranteed radial confinement of charged particles are destroyed by the addition of radial Ioffe fields.

One example is the radial field of a quadrupole Ioffe trap $\beta(x \hat{\mathbf{x}}-y \hat{\mathbf{y}})$, with $\beta$ determined by the geometry and current of the Ioffe trap. The field magnitude increases linearly with distance from the central axis, creating a field minimum that can radially confine a cold $\overline{\mathrm{H}}$ by its magnetic moment. Currents in four current bars [Fig. 2(a)] parallel to $B_{0} \hat{\mathbf{z}}$ produce such a field.

Higher-order Ioffe traps, produced using more current bars [e.g., Fig. 2(b)], offer smaller radial magnetic fields and gradients close to the center axis, lessening any gradient-related loss of $\bar{p}$ confined near this axis [19]. However, quadrupole Ioffe traps offer several potential advantages. First, deeper radial well depths for $\overline{\mathrm{H}}$ are possible, because the magnetic field magnitude for a quadrupole drops off much more slowly with increasing distance from the current bars than does that of a higher-order trap, in the region just inside the current bars where electrodes and support materials must be located. Second, enhanced decay-induced cooling [20] may be possible. Third, there is easier radial access for cooling lasers. Fourth, tighter $\overline{\mathrm{H}}$ confinement to the center axis optimizes overlap with axial cooling and spectroscopy lasers.

Without axial symmetry and guaranteed radial confinement, what particle loss should be expected? In the absence of $\bar{p}$ experiments, various answers have been offered. A Letter written to voice stability concerns [15] claimed that, in the absence of angular momentum conservation, adiabatic invariants could prevent radial loss of charged particles for low numbers and densities of charged particles, when a quadrupole Ioffe trap is added to a Penning trap. Resonances capable of causing particle loss seemed easily avoidable in the low density limit. As the particle density was increased, it was suggested that close collisions would eventually break these invariants and lead to particle loss, but the critical density could not be specified. A subsequent

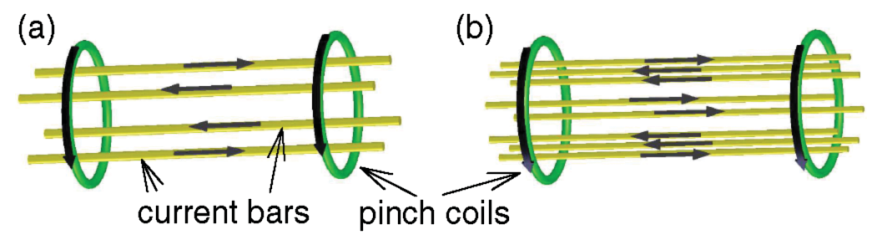

FIG. 2 (color). Current bars of simple (a) quadrupole and (b) octupole Ioffe traps are parallel to an externally produced bias magnetic field. Currents in pinch coils add an axial gradient.
Letter [16] claimed that the resonances could not be avoided, and the resulting diffusive loss of charge particles would "very likely $\ldots$ destroy the confinement of $\ldots e^{+}$ and $\bar{p}$." A follow-up study with electrons [17] encountered electron loss along magnetic field lines that was more severe than the predicted diffusive loss. The strong conclusion was that quadrupole Ioffe traps "cannot be used to trap antihydrogen ... in all currently described [antihydrogen production] schemes."

The $\bar{p}$ stability tests reported here use $\bar{p}$ from the unique Antiproton Decelerator (AD) of CERN. They are captured in the lower section of a series of 45 cylindrical gold-plated copper ring electrodes, each with a $R=1.8 \mathrm{~cm}$ inner radius. A spatially uniform magnetic field $B_{0}=1 \mathrm{~T}$ is directed along the symmetry axis of the rings. This relatively low field is needed to avoid reducing the well depth for the Ioffe trap, even though 4.5 times more $\bar{p}$ can be trapped per $\mathrm{AD}$ injection for $B_{0}>3 \mathrm{~T}$. Still, more than $30000 \bar{p}$ slow in a degrader, are captured in the Penning trap, and cool via collisions with cold electrons, for each $\bar{p}$ injection. These are photoelectrons liberated from a thin gold layer by $10 \mathrm{~ns}, 1.7 \mathrm{MW}$ excimer laser pulses at $248 \mathrm{~nm}$. More $\bar{p}$ are "stacked" if needed from successive $\mathrm{AD}$ injections [21]. The cooling electrons are released by removing the $\bar{p}$ trapping potential for a time short enough that they, but not the $\bar{p}$, can escape. For this test, the ejection was not optimized to ensure a $\bar{p}$ temperature near the $4.2 \mathrm{~K}$ of the apparatus.

The $\bar{p}$ are transferred through the series of cylindrical electrodes, from the place where they are initially electron cooled [Fig. 1(b)] to an electrode near the center of the deenergized quadrupole Ioffe trap [Fig. 1(b)], a move of $0.4 \mathrm{~m}$ through 18 electrodes. They are transferred adiabatically through one electrode at a time by manipulating the potentials applied to the electrodes. More $\bar{p}$ loss is detected during the transfer than expected, a likely consequence of an elevated $\bar{p}$ temperature. The $\bar{p}$ are held as long as desired. When released from the trap, their annihilations are counted as the $\bar{p}$ strike the degrader plate (used earlier to slow $\bar{p}$ for their initial capture). Four layers of scintillating fibers and two layers of plastic scintillator paddles detect the annihilations with a high efficiency, and counting coincidences from these detector layers reduces the background.

The radial field of the quadrupole Ioffe trap is produced by current in the superconducting current bars of racetrack coils (Fig. 1). Current sent through superconducting pinch coils would produce the axial gradient needed to axially confine $\overline{\mathrm{H}}$, adding to $B_{0}$ and making it depend upon $z$. Fields and critical currents for the magnetic trap were initially calculated at Harvard, where the cryogenic platform and Penning traps were designed and built. The final magnetic trap design, mechanical stability, and construction were the responsibility of the Jülich team [22]. Multistrand $\mathrm{NbTi}$ wire is wound on titanium forms. Strong outward forces after energization are contained by close fitting titanium parts and aluminum bands. The Ioffe 
trap is designed so that $69 \mathrm{~A}$ in the racetracks and $80 \mathrm{~A}$ in the pinch coils produce a gradient $\beta=93 \mathrm{~T} / \mathrm{m}$, a radialto-axial field ratio $\beta R / B_{0}=0.78$, and a $375 \mathrm{mK}$ well depth for ground-state $\overline{\mathrm{H}}$ in an external $1 \mathrm{~T}$ field. (The trap depth is $650 \mathrm{mK}$ without the $1 \mathrm{~T}$.) Four magnesium fluoride windows provide optical access for future $\overline{\mathrm{H}}$ laser cooling and spectroscopy, even at the ultraviolet $121.5 \mathrm{~nm}$ $\overline{\mathrm{H}}$ Ly $\alpha$ wavelength.

Figure 3(a) represents the trap electrodes within which the $\bar{p}$ are centered for the stability tests, with magnetic field lines of the radial Ioffe field and the bias field, along with equipotentials generated by applying $50 \mathrm{~V}$ between the electrodes. Field lines initially parallel to $\hat{\mathbf{z}}$ diverge to intersect electrodes when a strong Ioffe field is added. Figure 3(b) shows the well depth for $\bar{p}$ along various field lines, specified by their radial position at the center of the trap electrode $(z=0)$. The well depth increases slightly for field lines that are displaced radially from the trap center, out to an abrupt cutoff that occurs for field lines that intersect the center electrode. There is no potential well along such field lines. We would thus expect any $\bar{p}$ confined out at a larger radius to be lost when the Ioffe field of Fig. 3(a) is applied and that radial compression methods (not used in this work) could reduce $\bar{p}$ loss. Figures 3(c) and 3(d) pertain when the pinch coils are also energized. The useful trapping radius for $\bar{p}$ doubles as the pinch coils double the bias field. (a)
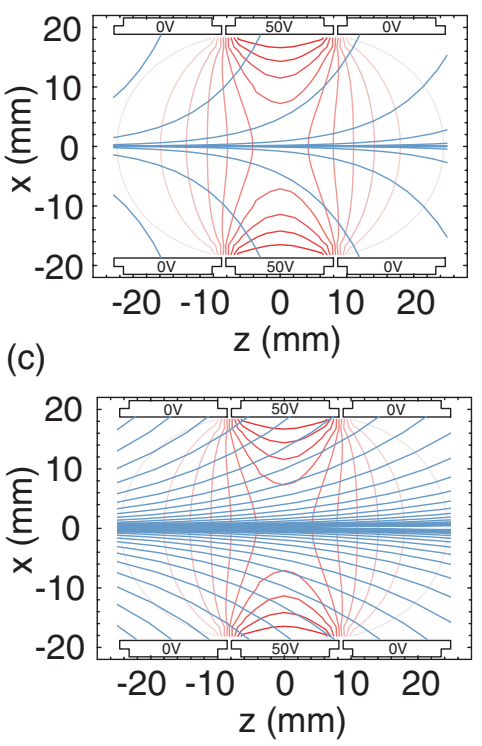

(b)
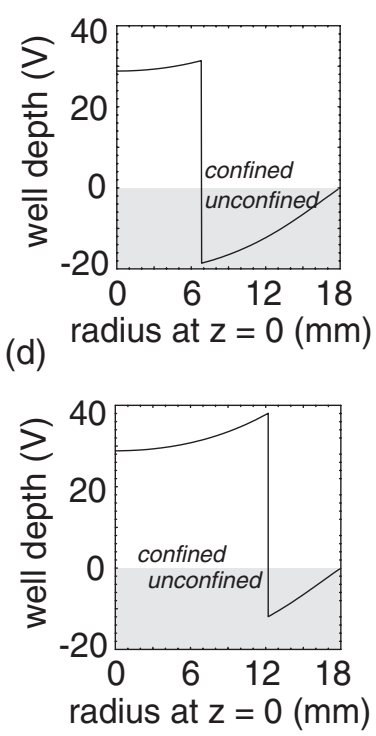

FIG. 3 (color). Cross section of cylindrical ring electrodes (black) in which $\bar{p}$ are stored, with superimposed magnetic field lines (blue) and electrostatic equipotentials in $5 \mathrm{~V}$ intervals (red). Magnetic field lines, (a) without and (c) with current in the pinch coils, diverge so as to intersect electrodes, even as they make a magnetic field whose magnitude increases with distance from the trap axis and center. The corresponding electrostatic well depths for a $\bar{p}$ moving along a field line are represented in (b) and (d).
To test for $\bar{p}$ stability, the radial magnetic field of a quadrupole Ioffe trap is applied after $\bar{p}$ have been centered within it. The Ioffe field is ramped up (over about ten minutes at $0.1 \mathrm{~A} / \mathrm{s}$ ), held constant for five minutes (enough time to make $\overline{\mathrm{H}}$ via the charge-exchange method), and then ramped back to zero, while $\bar{p}$ annihilation detectors monitor $\bar{p}$ loss. The surviving $\bar{p}$ are released from the trap and counted. Figure 4 shows that the fraction of $\bar{p}$ that survive a quadrupole Ioffe field decreases linearly with radial quadrupole current. The loss may be less if the axial $\bar{p}$ temperature is reduced, by optimizing the electron ejection, and/or by introducing cooling electrons into the $\bar{p}$ trap. Already, more $\bar{p}$ survive than needed to produce $\overline{\mathrm{H}}$ via charge exchange.

The other requirement for $\overline{\mathrm{H}}$ formation via charge exchange is that $e^{+}$must be confined near the trapped $\bar{p}$ and, hence, must also survive the radial field of a Ioffe trap. For a test, we substitute electrons which we expect to initially radiate synchrotron radiation to equilibrate at $4.2 \mathrm{~K}$. After loading and transferring $36 \times 10^{6}$ electrons into the center of the Ioffe trap, the radial Ioffe field is slowly ramped on, held constant, and then ramped off, just as described above for $\bar{p}$. As for $\bar{p}$, we expect that electrons outside of the cutoff radius of Fig. 3(b) will be lost. Still, the number of electrons remaining in the trap (Fig. 5) is more than the number of $e^{+}$needed for $\overline{\mathrm{H}}$ production via charge exchange, even at $\beta R / B_{0}=1$. The electron survival is substantially larger than observed earlier [17] for our highest radial fields, perhaps because of differences in unspecified electrostatic well depth and/or plasma temperature. The longer plasmas mostly used in the earlier study showed much larger losses.

Our principal focus, like that of the earlier Letters [1517], is on the effect of the radial quadrupole Ioffe field for two reasons. First, the axial Ioffe fields from pinch coils are axially symmetric. Second, it should be possible to reduce any $\bar{p}$ losses associated with these fields by relocating the pinch coils farther from the $\bar{p}$ trap. Still, the pinch coils will likely have some effect. For example, increasing the cur-

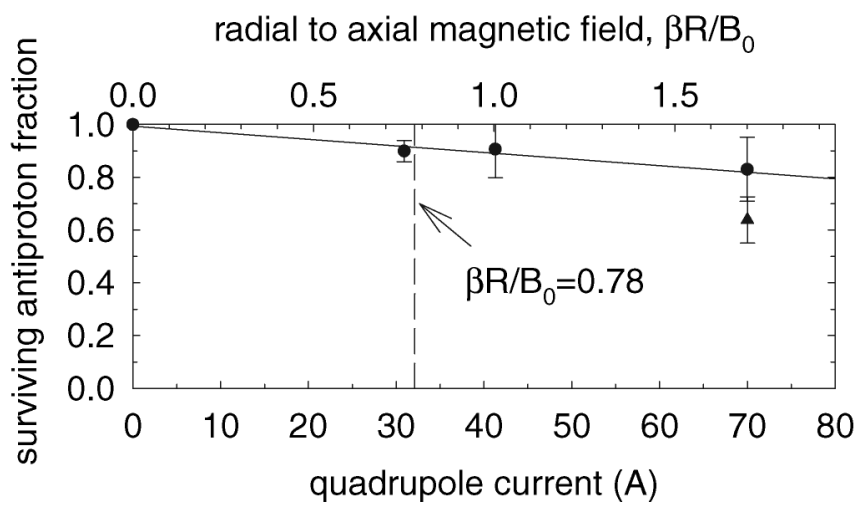

FIG. 4. Fraction of about 90000 (circles) and 280000 (triangle) trapped $\bar{p}$ that survive a radial Ioffe quadrupole field that is ramped up to a given current, held $300 \mathrm{~s}$, and ramped back down. 
radial to axial magnetic field, $\beta \mathrm{R}_{/} \mathrm{B}_{0}$

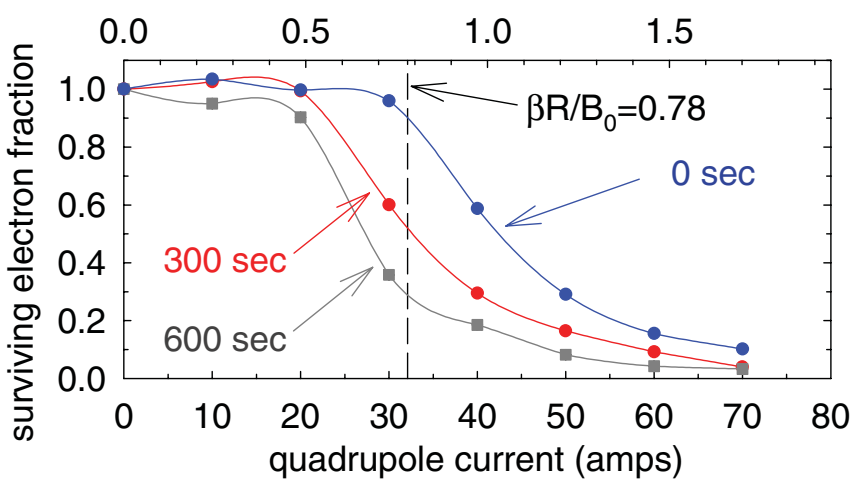

FIG. 5 (color). Fraction of electrons that survive a radial Ioffe quadrupole field that is ramped up to a given current, held 0,300, or $600 \mathrm{~s}$, and ramped back down.

rent in pinch coils should adiabatically increase the $\bar{p}$ cyclotron energy, raising the $\bar{p}$ temperature due to $\bar{p}-\bar{p}$ collisions. The effect of axial gradients must be studied experimentally when $\bar{p}$ are next available.

What else is required to trap $\overline{\mathrm{H}}$ atoms produced by charge exchange? First is an $\overline{\mathrm{H}}$ kinetic energy less than the trap depth (375 $\mathrm{mK}$ here). The energy of the $\overline{\mathrm{H}}$ is expected to be essentially that of the $\bar{p}$ from which they form, for this production method. For $\overline{\mathrm{H}}$ formed from $4 \mathrm{~K}$ $\bar{p}$, a small number of $\overline{\mathrm{H}}$ from the tail of a thermal distribution would have such low energies. Second, the $\overline{\mathrm{H}}$ states must have magnetic moments that are sufficiently large and properly oriented to be trapped. The moments of the highly excited $\overline{\mathrm{H}}$ atoms formed $[5,10]$ have not been measured, and it is not known if any ground-state atoms are produced. We remain intrigued by the possibility that some of the highly excited, highly polarizable $\overline{\mathrm{H}}$ states will be trapped [10] and remain so as radiation [20] slowly reduces the internal $\overline{\mathrm{H}}$ energy.

Finally, can a quadrupole Ioffe trap superimposed upon a nested Penning trap [6] be used to capture $\overline{\mathrm{H}}$ produced during positron cooling of antiprotons [7] in a nested Penning trap [6] - the most commonly used of the two methods to produce slow $\overline{\mathrm{H}}[7-10]$ ? A recent reinterpretation [23] of $\overline{\mathrm{H}}$ velocity measurements [24] suggests that $\overline{\mathrm{H}}$ atoms cold enough to trap may already be produced by this formation method. As a first step toward understanding whether such $\overline{\mathrm{H}}$ formation is compatible with a quadrupole Ioffe trap, we are encouraged that many $\bar{p}$ and electrons survive being moved adiabatically along the bias field direction within the radial Ioffe field for several centimeters. However, remaining stability questions await experimental answers when $\bar{p}$ are next available.

In conclusion, substantial numbers of $\bar{p}$ survive the effect of the radial magnetic field of a quadrupole Ioffe trap-enough to produce $\overline{\mathrm{H}}$ atoms within this field using the charge-exchange method for $\overline{\mathrm{H}}$ production. Substantial numbers of $e^{+}$can be stored in the appropriate location within the Ioffe field based upon a demonstration with electrons. Quadrupole Ioffe traps thus seem to have a role to play for $\overline{\mathrm{H}}$ formed by charge exchange, in spite of earlier claims to the contrary. Whether they have a role to play in trapping $\overline{\mathrm{H}}$ produced in a nested Penning trap remains to be seen.

We are grateful to CERN and the $\mathrm{AD}$ team for delivering the $5 \mathrm{MeV}$ energy antiprotons. This work was supported by the NSF and AFOSR of the U.S., the BMBF, DFG, and Jülich Laboratory of Germany, along with the NSERC, CRC, CFI, and OIT of Canada.

*Electronic address: gabrielse@ physics.harvard.edu

[1] G. Gabrielse, in Fundamental Symmetries, edited by P. Bloch, P. Pavlopoulos, and R. Klapisch (Plenum, New York, 1987), pp. 59-75.

[2] R. Bluhm, V. A. Kostelecký, and N. Russell, Phys. Rev. Lett. 82, 2254 (1999).

[3] G. Gabrielse, Hyperfine Interact. 44, 349 (1988).

[4] J. Walz and T. Hänsch, Gen. Relativ. Gravit. 36, 561 (2004).

[5] G. Gabrielse, Adv. At. Mol. Opt. Phys. 50, 155 (2005).

[6] G. Gabrielse, S.L. Rolston, L. Haarsma, and W. Kells, Phys. Lett. A 129, 38 (1988).

[7] G. Gabrielse et al., Phys. Lett. B 507, 1 (2001).

[8] M. Amoretti et al., Nature (London) 419, 456 (2002).

[9] G. Gabrielse et al., Phys. Rev. Lett. 89, 213401 (2002).

[10] G. Gabrielse et al., Phys. Rev. Lett. 89, 233401 (2002).

[11] E. A. Hessels, D. M. Homan, and M. J. Cavagnero, Phys. Rev. A 57, 1668 (1998).

[12] C. H. Storry et al., Phys. Rev. Lett. 93, 263401 (2004).

[13] R. van Roijen, J. J. Berkhout, S. Jaakola, and J. T. M. Walraven, Phys. Rev. Lett. 61, 931 (1988).

[14] C. L. Cesar, D. G. Fried, T. C. Killian, A. D. Polcyn, J. C. Sandberg, I. A. Yu, T. J. Greytak, D. Kleppner, and J. M. Doyle, Phys. Rev. Lett. 77, 255 (1996).

[15] T. M. Squires, P. Yesley, and G. Gabrielse, Phys. Rev. Lett. 86, 5266 (2001).

[16] E. P. Gilson and J. Fajans, Phys. Rev. Lett. 90, 015001 (2003).

[17] J. Fajans, W. Bertsche, K. Burke, S. F. Chapman, and D. P. van der Werf, Phys. Rev. Lett. 95, 155001 (2005).

[18] T. M. O’Neil, Phys. Fluids 23, 2216 (1980).

[19] J. Fajans and A. Schmidt, Nucl. Instrum. Methods Phys. Res., Sect. A 521, 318 (2004).

[20] T. Pohl, H. R. Sadeghpour, Y. Nagata, and Y. Yamazaki, Phys. Rev. Lett. 97, 213001 (2006); C. L. Taylor, J. Zhang, and F. Robicheaux, J. Phys. B 39, 4945 (2006).

[21] G. Gabrielse et al., Phys. Lett. B 548, 140 (2002).

[22] The team was working in close cooperation with the ACCEL company.

[23] T. Pohl, H. R. Sadeghpour, and G. Gabrielse, Phys. Rev. Lett. 97, 143401 (2006).

[24] G. Gabrielse et al., Phys. Rev. Lett. 93, 073401 (2004). 